\section{A violência familiar e a criança e o adolescente com deficiências}

\author{
Family violence in a sample of children and \\ adolescents with disabilities
}

\section{La violencia familiar y el niño y/o adolescente con discapacidad}

\section{Resumo}

O trabalho objetivou identificar e analisar a prevalência da violência familiar física e psicológica entre crianças e adolescentes com diferentes categorias de deficiência em um hospital no Rio de Janeiro, Brasil. Estudo observacional, transversal realizado com aplicação do instrumento Parent-Child Conflicts Tatics Scales numa amostra de 270 responsáveis. Mostrou-se a prevalência de 83,7\% para agressão psicológica e 84,4\% para maus-tratos físicos, e 96,5\% das crianças e dos adolescentes com deficiência que sofreram punição corporal, também foram vítimas de agressão psicológica $(p<0,01)$, e todos os que sofreram maus-tratos físicos graves sofreram agressão psicológica $(p=0,01)$. Crianças e adolescentes com deficiência apresentam maior risco de sofrer violência intrafamiliar do que aquelas sem deficiência. Conclui-se haver necessidade de maior conscientização e capacitação das equipes de saúde em relação à detecção e notificação dos casos de maus-tratos da população estudada e para as medidas de proteção, e esforços devem ser feitos para apoiar essas famílias.

Violência Doméstica; Maus-Tratos Infantis; Crianças com Deficiência; Adolescente
Ana Cláudia Mamede Wiering de Barros ${ }^{1}$ Suely Ferreira Deslandes 1

Olga Maria Bastos 1 


\section{Introdução}

A violência familiar pode causar variados agravos físicos e mentais, apresenta alta prevalência e pelos danos que provoca é reconhecida pela Organização Mundial da Saúde (OMS) como importante problema de saúde pública. Mulheres, idosos, crianças e adolescentes apresentam maior suscetibilidade a esse tipo de violência 1,2,3. Dentro desse contexto, aqueles que necessitam de cuidados complexos e contínuos, como crianças e adolescentes com deficiência, encontramse ainda mais vulneráveis às diversas formas de maus-tratos (físicos, psicológicos, negligência e abuso sexual) 4,5,6,7,8.

Não existe no Brasil publicação recente de inquéritos nacionais sobre a prevalência da violência familiar em relação à criança e ao adolescente. Mesmo dentre os países desenvolvidos, há um nível desigual no investimento da notificação desses casos de violência. Assim, mesmo com a gradativa implantação de uma ficha de notificação compulsória no âmbito do Sistema Único de Saúde (SUS) a ser adotada em todas as unidades do país, as estatísticas oficiais ainda subestimam o fenômeno 9,10 .

A deficiência é considerada como uma condição crônica de saúde que se manifesta por um tempo prolongado ou indefinido e que necessita de cuidados de saúde especiais e muitas vezes complexos 4,11. São descritos quatro tipos de deficiência: deficiência física, deficiência sensorial, deficiência intelectual e múltiplas deficiências 12 .

Pesquisas internacionais revelam que crianças e adolescentes com quaisquer dos tipos de deficiência encontram-se mais vulneráveis à violência familiar do que crianças e adolescentes sem deficiência 2,7,13. Diversos trabalhos mostram que dependendo do tipo de deficiência apresentada pela criança ou pelo adolescente, ocorrerá uma maior prevalência de determinado tipo de maus-tratos. Estudos 8,14,15 apontam que crianças com deficiência que têm problemas de comportamento são as que apresentam maior vulnerabilidade à violência, quando comparadas às crianças sem deficiência: 7 vezes para negligência, violência física e violência psicológica, e 5,5 vezes para abuso sexual. Por outro lado, as que tinham deficiência múltipla mostraram vulnerabilidade maior para violência física e abuso sexual do que as crianças que tinham apenas um tipo de deficiência.

Dada a escassez de estudos científicos brasileiros sobre violência e deficiência, este trabalho se propôs a identificar e a analisar a prevalência da violência familiar física e psicológica entre crianças e adolescentes com diferentes categorias de deficiência (física, intelectual e múltipla), atendidos em um hospital da rede pública do Rio de Janeiro, Brasil.

\section{Metodologia}

Foi realizado um estudo observacional, transversal, exploratório, no período de julho de 2012 a fevereiro de 2013, em um hospital federal no Município do Rio de Janeiro, que oferece atendimento terciário em diversas especialidades, dentre elas, Neurologia, Neurocirurgia, Genética, Pediatria e Adolescência. Essas especialidades atendem crianças e adolescentes com deficiência encaminhados de vários municípios do Estado do Rio de Janeiro, por serem referência em suas áreas de atuação.

Os sujeitos de pesquisa foram familiares responsáveis (mães, avós e pais) pelos cuidados cotidianos de crianças e adolescentes com deficiência física, intelectual e múltipla, atendidos nesses ambulatórios, cuja população assistida mensalmente se encontra em torno de 450 crianças e adolescentes. A escolha dos serviços de Pediatria e de Adolescentes para captação dos sujeitos de pesquisa se deu por haver pacientes com deficiência que são atendidos exclusivamente neste serviço e que fazem o acompanhamento neurológico e/ou de genética em outros hospitais.

Foram incluídos no estudo os responsáveis por crianças a partir de 12 meses de idade (para que houvesse um tempo mínimo de convivência pais/avós/filho(a)/neto(a)) até 21 anos, que tivessem deficiência física, intelectual ou múltipla. Aqueles responsáveis por crianças e adolescentes que apresentaram deficiência sensorial foram excluídos do estudo, por ser pequeno o número de pacientes atendidos nesse hospital com tal tipo de deficiência, o que levaria a uma amostra estatisticamente insignificante. Também foram excluídos da pesquisa aqueles responsáveis que apresentaram déficit cognitivo, o que dificultaria o entendimento das perguntas do questionário. Os responsáveis eram convidados a participar do estudo, após confirmado serem eles os que cuidavam da criança ou do adolescente no dia a dia, não apenas os trazendo para a consulta.

Foi aplicado um questionário adaptado transculturalmente e validado no Brasil, o $\mathrm{Pa}$ rent-Child Conflict Tactics Scales (CTSPC) 16,17 em responsáveis por crianças e adolescentes com deficiência. A utilização do instrumento serviu para estimar a prevalência de violência familiar dentre essas crianças e adolescentes, identificando a que tipo de violência eram submetidos.

O instrumento CTSPC tem como objetivo, mensurar maus-tratos físicos e psicológicos na relação pais-crianças ou adolescentes, bem 
como os modelos não violentos de disciplina, classificando as respostas como: disciplina não violenta, agressão psicológica e maus-tratos físicos. Os maus-tratos físicos são divididos em três grupos de acordo com a gravidade da agressão: punição corporal, maus-tratos físicos graves, e maus-tratos físicos gravíssimos. Os itens relacionados à agressão física diferem em relação à gravidade, e a escala foi desenvolvida para levar em conta essa importante diferença. Assim sendo, a pontuação dos diferentes tipos de agressão física variou de acordo com a seriedade do ato, e os mais graves tiveram escores mais altos. Em relação à cronicidade da agressão, o questionário apresenta as seguintes opções: nunca, raramente ( 1 a 3 vezes ao ano), às vezes (4 a 10 vezes ao ano), e muitas vezes (10 ou mais vezes ao ano) 16,18 .

Pela aplicação do instrumento CTSPC, buscou-se avaliar a frequência de situações de maustratos físicos e psicológicos ocorridos nos 12 meses anteriores à entrevista.

Uma equipe treinada, composta por seis pesquisadores nas áreas da Medicina, Psicologia e Sociologia, que desconhecia a história pregressa dos sujeitos de pesquisa, aplicou o instrumento CTSPC. Os questionários foram lidos, para que não houvesse perda no número de entrevistados, já que alguns deles poderiam ter dificuldade de leitura. A pesquisa foi feita na área do hospital destinada à espera do atendimento, aproveitando que o responsável aguardava a consulta do seu/sua filho(a)/neto(a). Escolhemos um local mais afastado e silencioso para aplicação do instrumento. Não houve recusa e mais de uma vez os responsáveis se ofereceram para participar da pesquisa.

Para o cálculo amostral, definiram-se os seguintes parâmetros: uma prevalência de violência de $50 \%$ que estimou o maior tamanho amostral possível, já que não existem estudos nacionais que definam a prevalência de violência contra as pessoas com deficiência; um erro amostral de $5 \%$ e um nível de 0,05 de confiança $(\alpha)$. Considerando que são atendidos mensalmente 450 crianças e adolescentes com deficiência nos ambulatórios de Neurologia, Neurocirurgia, Genética, Pediatria e Adolescente, a amostra calculada definiu 270 responsáveis a serem entrevistados.

As variáveis consideradas no estudo foram: sexo e idade da criança e do adolescente; grau de instrução dos responsáveis (Associação Brasileira de Empresas de Pesquisa. Critérios de Classificação Econômica Brasil. http://www.abep.org/ new/Serviços/Download.aspx?id=01); tipos de violências; tipos de deficiência. A associação da prevalência da violência e do tipo de violência com outras características da população estu- dada foi avaliada pelo teste qui-quadrado (em relação à disciplina não violenta, agressão psicológica, punição corporal e maus-tratos físicos graves), pelo teste de Fisher (em relação à maustratos físicos gravíssimos) e pelo cálculo da razão de prevalência (RP), com respectivo intervalo de 95\% de confiança (IC95\%). O nível de significância do estudo foi 0,05. Para o processamento e a análise de dados, foi utilizado programa Epi Info 7.0 (Centers for Disease Control and Prevention, Atlanta, Estados Unidos). Esta pesquisa foi aprovada pelo Comitê de Ética em Pesquisa do hospital onde o estudo foi realizado sob o C.A.E número 079521126.0000.5269.

\section{Resultados}

Dos 270 questionários aplicados aos responsáveis pelas crianças e adolescentes com deficiência, foi observado um predomínio da deficiência intelectual em 115 pacientes $(42,6 \%)$, cuja principal causa foi a síndrome de Down, seguidos por 91 pacientes $(33,7 \%)$ com deficiência múltipla e $64(23,7 \%)$ com deficiência física.

Em relação às características sociodemográficas, 136 (50,4\%) eram meninos e 134 (49,6\%) meninas. A idade variou de 1 ano e 2 meses a 20 anos e 3 meses, com média de 10,7 anos e desvio padrão de $\pm 5,33$. O extrato social predominante das famílias foi a classe C (C1 com $37 \%$ e C2 com 29\%). A escolaridade dos responsáveis pelas crianças e adolescentes com deficiência apresentou distribuição homogênea em relação aos diferentes níveis, sendo observado que $42 \%$ deles tinham pelo menos o ensino médio.

Em relação aos diferentes tipos de violência intrafamiliar, o estudo encontrou uma prevalência de $83,7 \%$ para agressão psicológica e $84,4 \%$ de maus-tratos físicos e dentre eles, segundo a classificação de Straus, $74 \%$ para punição corporal, $9,6 \%$ para maus-tratos físicos graves e 2,2\% para maus-tratos físicos gravíssimos.

Verificou-se que apenas $11,1 \%$ dos responsáveis relataram usar apenas de técnicas não violentas para a resolução dos conflitos entre eles e seus filhos(as)/netos(as). Trinta e nove crianças e adolescentes (14,4\%) foram vítimas de um tipo de violência, enquanto $42 \%$ de dois tipos e $32 \%$ de três ou mais tipos de agravo.

A análise dos dados mostrou não haver diferença estatisticamente significativa entre o perfil de violência, quando comparado o sexo masculino com o feminino ( $\mathrm{p}=0,134)$, embora, quando analisado isoladamente em relação à punição corporal, tenha se encontra do uma frequência maior em meninos ( $\mathrm{p}=0,008$; RP = 1,22; IC95\%: $1,05-1,43)$. 
Quando observados os diferentes tipos de violência em relação aos tipos de deficiência, os resultados apontam uma prevalência maior de maus-tratos físicos e psicológicos em crianças e adolescentes com deficiência intelectual, quando comparados aos outros tipos de deficiência (Tabela 1). Apenas punição corporal sofreu influência da idade, mostrando que a criança entre três e cinco anos (pré-escolar) sofria mais esse agravo quando comparada ao lactente (até dois anos de idade) ( $\mathrm{p}=0,008$; RR $=1,87$; IC95\%: 1,08-3,23).

A frequência dos distintos tipos de violência não apresentou diferença significativa segundo o grau de instrução dos responsáveis ao comparar cada nível de escolaridade com o nível superior completo, que é considerado o grau de instrução menos vulnerável à prática de maus-tratos 19,20.

Ao analisar os diferentes tipos de maus-tratos pesquisados e a relação entre eles, identificou-se que $96,5 \%$ das crianças e dos adolescentes com deficiência que sofreram punição corporal também foram vítimas de agressão psicológica ( $\mathrm{p}<$ 0,01; RP = 5,24; IC95\%: 2,65-10,36), e que todos os que sofreram maus-tratos físicos graves também sofreram agressão psicológica ( $\mathrm{p}=0,01$ ).

\section{Discussão}

Os resultados desta pesquisa evidenciaram alta prevalência de violência familiar entre crianças e adolescentes com deficiência; cerca de $75 \%$ foi vítima de dois ou mais tipos diferentes de abuso, corroborando os estudos internacionais $7,8,14,19$. Quando múltiplas formas de maus-tratos são verificadas, a violência física é a segunda causa mais prevalente de abuso e a violência psicológica, a primeira. Usualmente, a agressão psicológica vem acompanhada por outro tipo de maus-tratos 6,13,21. Embora não seja possível dizer neste estudo que tipo de violência ocorreu primeiro, a literatura mostra que a violência psicológica geralmente precede à física, muitas vezes não sendo identificada, pelo fato de os responsáveis não a reconhecerem como um tipo de maus-tratos 22,23.

A alta prevalência de punição corporal encontrada neste estudo pode estar relacionada ao entendimento por parte dos responsáveis de que estão utilizando uma tática educativa. Segundo a OMS 6 e também demonstrado em estudos, os responsáveis frequentemente confundem punição com disciplina, usando a força física para a correção ou mudança de comportamento das crianças e dos adolescentes, independentemente de eles terem ou não deficiência 24,25,26. Esta pesquisa mostrou ainda que meninos com deficiência apresentam maior prevalência para os maustratos físicos do que as meninas com deficiência, o que se verifica na literatura internacional 6,14.

Em relação à idade das crianças e dos adolescentes com deficiência e o tipo de violência sofrida, houve associação entre crianças no nível pré-escolar e punição corporal. Segundo Sullivan 6, crianças com deficiência são maltratadas mais precocemente do que aquelas sem deficiência, sendo a faixa etária de até 5 anos a mais acometida.

Em relação aos tipos de disciplina utilizados pelos responsáveis para a resolução de conflitos com seus(suas) filhos(as)/netos(as), nota-se uma alta prevalência para o uso de táticas não violentas, ao mesmo tempo em que também se encontra uma alta prevalência de práticas violentas. Isso evidencia, conforme confirma o estudo de Hendricks et al. 8 que embora esses responsáveis conheçam e exerçam técnicas de disciplina não

Tabela 1

Tipos de disciplina (violenta ou não) em relação a cada categoria de deficiência estudada em crianças e adolescentes com deficiência atendidos em um hospital terciário.

\begin{tabular}{|c|c|c|c|c|}
\hline \multirow[t]{3}{*}{ Tipo de disciplina } & \multicolumn{3}{|c|}{ Tipo de deficiência } & \multirow[t]{3}{*}{ Valor de $\mathrm{p}$} \\
\hline & Física (\%) & Intelectual (\%) & Múltipla (\%) & \\
\hline & {$[n=64]$} & {$[n=115]$} & {$[\mathrm{n}=91]$} & \\
\hline Disciplina não violenta & $63(98,4)$ & $113(98,2)$ & $76(83,5)$ & * \\
\hline Agressão psicológica & $53(82,8)$ & $105(91,3)$ & $68(74,7)$ & 0,0005 \\
\hline Punição corporal & $42(65,6)$ & $96(83,4)$ & $58(63,7)$ & 0,04 \\
\hline Maus-tratos físicos graves & $7(10,9)$ & $12(10,4)$ & $7(7,7)$ & 1,73 \\
\hline Maus-tratos físicos gravíssimos & $1(1,5)$ & $4(3,5)$ & $1(1,1)$ & * \\
\hline
\end{tabular}

* Teste de qui-quadrado não aplicado, devido ao \% das caselas apresentar valores $<5$. 
violenta, quando consideram que somente isso não basta para que a criança ou o adolescente obedeça, eles também usam de violência para a resolução das desavenças familiares.

Nesta pesquisa foi possível afirmar que crianças e adolescentes com deficiência intelectual sofreram mais punição corporal e agressão psicológica do que as crianças e adolescentes com deficiência física ou múltipla. Estudos internacionais confirmam esse achado, indicando que as crianças com deficiência intelectual, problemas de comunicação ou problema de comportamento são as mais maltratadas, com oito vezes mais probabilidade de sofrerem abuso psicológico do que crianças com outro tipo de deficiência $4,8,20$. Neste trabalho, entretanto, encontrou-se uma forte associação entre punição corporal e maus-tratos físicos graves com agressão psicológica, em que praticamente todas as crianças que sofreram os dois tipos de maus-tratos físicos também sofreram agressão psicológica. As questões que podem estar relacionadas a essa maior vulnerabilidade seriam: a dificuldade em se comunicar; não ser dado crédito ao seu relato; a insegurança emocional; a frustração dos responsáveis por não obterem o comportamento esperado pelo fato de o(a) filho(a)/neto(a) apresentar problemas cognitivos 4,8,11,27,28.

Uma limitação deste estudo diz respeito à possibilidade de a amostra calculada não constituir uma amostra aleatória, pois essa população foi definida pelo tipo de atendimento especializado que é oferecido no hospital em questão. Portanto, essa amostra pode ser definida como uma amostra de conveniência.

Outra limitação foi o fato de não terem sido incluídos nesta pesquisa casos de deficiência sensorial, que é considerada como a mais prevalente nos dados divulgados no último Censo Demográfico, realizado em 2010 (Instituto Brasileiro de Geografia e Estatística. Censo Demográfico 2010. http://censo2010.ibge.gov.br/).

\section{Conclusões}

Considerando-se que as crianças e adolescentes com deficiência mostraram ter alta prevalência de sofrer violência familiar, há de se refletir sobre as especificidades de abordagem de tais casos. $\mathrm{O}$ reconhecimento dos sinais de maus-tratos entre crianças e adolescentes com deficiência intelectual pode ser dificultado pela incapacidade que eles têm de verbalizar que estão sendo maltratados, ou por não compreenderem que estão sendo agredidos. Naqueles com deficiência física, a dificuldade em falar sobre a violência sofrida pode estar associada ao fato de quem agride é o mesmo que cuida, havendo elos afetivos, além do receio do abandono após a identificação de maus-tratos.

No que diz respeito às equipes de saúde, observa-se a necessidade de maior conscientização e capacitação delas em relação à detecção e notificação dos casos de maus-tratos da população estudada. Esforços concretos precisam ser feitos para apoiar famílias com crianças e adolescentes com deficiência. Ter um(a) filho(a)/neto(a) com deficiência representa uma carga maior de cuidados, de gastos, de tempo mobilizado. Crianças e adolescentes deficientes muitas vezes não correspondem às expectativas dos pais, que planejaram um modelo de filho ideal, podendo levar a um quadro de depressão materna, rejeição e desenvolvimento de expectativas além das que a criança e o adolescente possam oferecer. Como consequência, tais pais não conseguem fazer um vínculo positivo com eles, veem-se isolados e sem suporte social ou de políticas públicas adequadas. Isso inegavelmente pode contribuir para maior estresse e diminuir a tolerância no trato cotidiano.

Os profissionais de saúde, por sua vez, precisam de estar atentos aos sinais de violência, identificando-os e, conforme preconiza o Estatuto da Criança e Adolescente, notificando ao Conselho Tutelar, ou outro órgão competente na ausência desse, para que as medidas de proteção cabíveis e demandadas ao Sistema de Garantia de Direitos das Crianças e Adolescentes sejam realizadas e para que possam também dar suporte às famílias. 


\section{Colaboradores}

A. C. M. W. Barros contribuiu na pesquisa bibliográfica; aplicação dos questionários; elaboração e redação do artigo. S. F. Deslandes colaborou na análise e interpretação dos dados; orientação metodológica; revisão do artigo. O. M. Bastos contribuiu na aplicação dos questionários; orientação metodológica; e revisão crítica do artigo.

\section{Referências}

1. United Nations Childen's Fund. Violence against disabled children. Findings and recommendations. New York: United Nations Childen's Fund; 2005.

2. World Health Organization; International Society for Prevention of Child Abuse and Neglect. Preventing child maltreatment: a guide to taking action and generating evidence. Geneva: World Health Organization; 2006.

3. World Health Organization. World report on disabilities. Geneva: World Health Organization 2011.

4. Gonzalvo GO. Maltrato en niños con discapacidades: características y factores de riesgo. An Esp Pediatr 2002; 56:219-23.

5. Manders JE, Stoneman Z. Children with disabilities in child protective services system: an analog study of investigation and case management. Child Abuse Negl 2009; 33:229-37.

6. Sullivan PM. Violence exposure among children with disabilities. Clin Child Fam Psychol Rev 2009; 12:196-216.

7. Jones L, Bellis M, Wood S, Hughes K, McCoy E, Eckley L, et al. Prevalence and risk of violence against children with disabilities: a systematic review and meta-analysis of observational studies. Lancet 2012; 380:899-907.

8. Hendricks C, Lansford J, Deater-Deckard K, Bornstein $\mathrm{MH}$. Associations between child disabilities and caregiver discipline and violence in low and middle-income countries. Child Dev 2014; 85 : 513-31.

9. Pinheiro PS. World report on violence against children. Geneva: World Health Organization; 2006.

10. Rates SMM, Melo EM, Mascarenhas MDM, Malta DC. Violência infantil: uma análise das notificações compulsórias, Brasil 2011. Ciênc Saúde Coletiva 2015; 20:655-65.

11. American Academy of Pediatrics. Committee on Child Abuse and Neglect and Committee on Children with Disabilities. Assessment of maltreatment of children with disabilities. Pediatrics 2001; 108:508-12.
12. Assis SG. Atenção a pessoas em situação de violência sob as perspectivas do ciclo de vida e das vulnerabilidades. Rio de Janeiro: Programa de Educação à Distância, Escola Nacional de Saúde Pública Sergio Arouca, Fundação Oswaldo Cruz; 2007.

13. Sullivan PM, Knutson JF. The association between child maltreatment and disabilities in a hospitalbased epidemiological study. Child Abuse Negl 1998; 22:271-88.

14. Sullivan PM, Knutson JF. Maltreatment and disabilities: a population-based epidemiological study. Child Abuse Negl 2000; 24:1273-2000.

15. Hibbard RA, Desch LW; American Academy of Pediatrics Committee on Child Abuse and Neglect and Council on Children with disabilities. Maltreatment of children with Disabilities. Pediatrics 2007; 119:1018-25.

16. Straus MA, Hamby SL. Measuring physical and psychological maltreatment of children with the conflict tactics scales. In: Kaufman KG, Jasinski JL, editors. Out of the darkness: contemporary perspectives on family violence. Thousand Oaks: Sage Publications; 1997. p. 119-35.

17. Reichenheim ME, Moraes CL. Adaptação transcultural do instrumento Parent-Child Conflict Tactics Scales (CTSPC) utilizado para identificar a violência contra a criança. Cad Saúde Pública 2003; 19: 1701-12.

18. Straus MA, Hamby SL, Finkelhor D, Moore DW, Runyan D. Identification of child maltreatment with the Parent-Child Conflict Tactics Scales: development and psychometric data for a national sample of american parent. Child Abuse Negl 1998; 22:249-70

19. Goldson E. Maltreatment among children with disabilities. Infants Young Child 2001; 13:44-54.

20. Murphy N. Maltreatment of children with disabilities: the breaking point. J Child Neurol 2011; 26:1054-6.

21. LeRoy M, Mahoney A, Boxer P, Gullan RL, Fang Q. Parents who hit and scream: interactive effects of verbal and severe physical aggression on clinic-referred adolescents' adjustment. Child Abuse Negl 2014; 38:893-901. 
22. Silva LL, Coelho EBS, Caponi SNC. Violência silenciosa: violência psicológica como condição da violência física doméstica. Interface Comun Saúde Educ 2007; 11:93-103.

23. Rees CA. Understanding emotional abuse. Arch Dis Child 2010; 95:59-67.

24. Weber LND, Viezzer AP, Brandenburg OJ. O uso das palmadas e surras como prática educativa. Estud Psicol (Natal) 2004; 9:227-37.

25. Holden GW, Brown AS, Baldwin AS, Caderao KC. Research findings can change attitudes about corporal punishment. Child Abuse Negl 2014; 38: 902-8.

\section{Abstract}

This study aimed to identify and analyze the prevalence of physical and psychological family violence in a sample of children and adolescents with different categories of disabilities in a hospital in Rio de Janeiro, Brazil. This was a cross-sectional observational study based on application of the Parent-Child Conflict Tactics Scale in a sample of 270 parents or guardians. Prevalence was $83.7 \%$ for psychological aggression and $84.4 \%$ for physical maltreatment, while $96.5 \%$ of the children and adolescents with disabilities that suffered physical punishment were also victims of psychological aggression $(p<0.01)$ and $100 \%$ of those who suffered severe physical maltreatment also suffered psychological aggression $(p=0.01)$. Children and adolescents with disabilities showed increased risk of suffering family violence compared to those without disabilities. In conclusion, there is a need for greater awareness-raising and training of health teams for detection and notification of child abuse, and efforts should be made to support these families.

Domestic Violence; Child Abuse; Disabled Children; Adolescent
26. Ho GWK, Gross DA. Differentiating physical discipline from abuse: $Q$ findings from Chinese American mothers and pediatric nurses. Child Abuse Negl 2015; 43:83-94.

27. Cavalcante FG, Bastos OM. Pessoas portadoras de deficiência e com necessidades especiais e situações de violência. In: Njaine K, Assis SG, Constantino P, organizadoras. Impactos da violência na saúde. Rio de Janeiro: Editora Fiocruz; 2009. p. 259-87.

28. Svenson B, Eriksson UB, Janson S. Exploring risk for abuse of children with chronic conditions or disabilities - parent's perceptions of stressors and the role of professionals. Child Care Health Dev 2013; 39:887-93.

\section{Resumen}

El trabajo tuvo como objetivo identificar y analizar la prevalencia de la violencia familiar física y psicológica entre niños y adolescentes con diferentes categorías de discapacidad en un hospital de Río de Janeiro, Brasil. Se trata de un estudio observacional, transversal, realizado a partir de la aplicación de la herramienta Parent-Child Conflict Tactics Scale en una muestra de 270 responsables. Se mostró una prevalencia de un $83,7 \%$ en el caso de la agresión psicológica y un $84,4 \%$ en el de malos tratos físicos, siendo que un $96,5 \%$ de los niños y de los adolescentes con discapacidad que sufrieron castigo corporal, también fueron víctimas de agresión psicológica $(p<0,01)$ y todos los que sufrieron malos tratos físicos graves sufrieron agresión psicológica $(p=0,01)$. Niños y adolescentes con discapacidad presentan un mayor riesgo de sufrir violencia intrafamiliar que aquellos sin discapacidad. Se concluye que existe necesidad de una mayor concienciación y capacitación de los equipos de salud, en relación con la detección y notificación de los casos de malos tratos de la población estudiada y desarrollo de medidas de protección y esfuerzos en apoyo a estas familias.

Violencia Doméstica; Maltrato a los Niños; Niños con Discapacidad; Adolescente
Recebido em 08/Jun/2015

Versão final reapresentada em 17/Ago/2015 Aprovado em 18/Fev/2016 